\title{
Finite Element Simulation of Lamb Wave Detection in Thin Plate
}

\author{
Chun'an Ai, Xiaofeng Cai, Jian Li, Kaixuan Liu \\ 601 Staff Room, Xi'an High-tech Institute, Xi'an, 710025, China
}

Keywords: Lamb wave, FEM, sound field, thin plate.

\begin{abstract}
In order to reveal the Lamb wave propagation mechanism, the Lamb wave detection of thin plate structure was investigated numerically. To this end, a finite element model was established and the corresponding boundary conditions, mesh size and time step were set. On this basis, the displacement field was solved and the Lamb wave mode was determined in single plate. Compared with the frequency dispersion curve, the error of numerical results is $1.3 \%$, which proves the validity of the numerical results. In addition, for the bilayer plate, the propagation of the Lamb wave between two mediums could be observed by solving the sound field at different times.
\end{abstract}

\section{Introduction}

Now ultrasonic testing is applied in many manufacturers to detect the metallurgical defects like inclusions, process defects like cracks and tissue defects. Through this way, substandard products are eliminated in time [1]. Compared with conventional ultrasonic testing, Lamb wave is used in a long distance and large range detection. But the propagation mechanism of Lamb wave in the plate is very complex, and the interaction between wave and defects is not very clear. At present, many scholars have carried out a lot of research on the Lamb wave detection of the thin plate, but it is mainly from the experimental view, which can't fundamentally reveal the propagation and scattering mechanism of Lamb wave [2]. Therefore, numerical simulation of Lamb wave is very important, it can not only enhance the comprehension on ultrasonic echo and ultrasonic imaging [3], but also be more flexible and superior in terms of modeling and parameters selection compared to the physical objects [4].

\section{Finite element equation in the thin plate}

Finite element method (FEM) is a common numerical method which is used to solve the field problem. The main advantage of FEM is unlimited to shape, boundary conditions and material properties. Therefore, the method can be introduced into the numerical calculation of sound field.

Assuming the thin plate is elastic, the center of a micro-element moves from the point $P(x, y, z)$ to the point $P^{\prime}(\bar{x}, \bar{y}, \bar{z})$.

The displacement components are as follows:

$$
u=\bar{x}-x, v=\bar{y}-y, W=\bar{z}-z
$$

The strain components are as follows:

$$
\begin{aligned}
& \varepsilon_{x x}=\frac{\partial u}{\partial x}, \varepsilon_{y y}=\frac{\partial v}{\partial y}, \varepsilon_{x x}=\frac{\partial w}{\partial z} \\
& \gamma_{y z}=\frac{\partial w}{\partial y}+\frac{\partial v}{\partial z}, \gamma_{z x}=\frac{\partial u}{\partial z}+\frac{\partial w}{\partial x}, \gamma_{x y}=\frac{\partial v}{\partial x}+\frac{\partial u}{\partial y}
\end{aligned}
$$

Fig. 1 shows the micro-element, the sides length of which are $d x, d y, d z$. The balance equations are as follows: 
$\frac{\partial \sigma_{x}}{\partial x}+\frac{\partial \tau_{y x}}{\partial y}+\frac{\partial \tau_{z x}}{\partial z}+f_{x}=0$

$\frac{\partial \tau_{x y}}{\partial x}+\frac{\partial \sigma_{y}}{\partial y}+\frac{\partial \tau_{z y}}{\partial z}+f_{y}=0$

$\frac{\partial \tau_{x z}}{\partial x}+\frac{\partial \tau_{y z}}{\partial y}+\frac{\partial \sigma_{z}}{\partial z}+f_{z}=0$

$\sigma, \tau, f$ represent the normal stress, shear stress and force density.

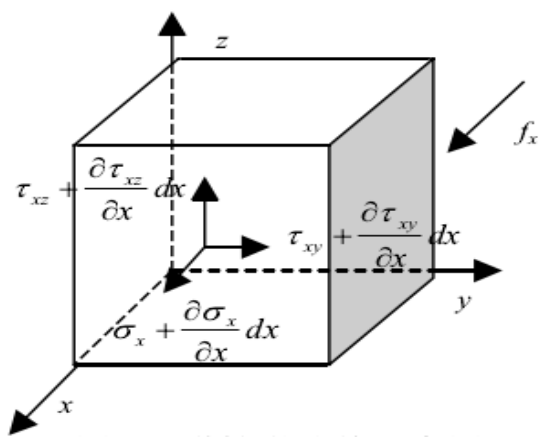

Fig. 1 Micro element

The stress-strain relationship is shown in the tensor form:

$\sigma=C \varepsilon$

$C, \sigma, \varepsilon$ represent the stiffness tensor, stress and strain tensor. Also they can be expressed in the array form:

$$
\sigma=\left\{\begin{array}{l}
\sigma_{x} \\
\sigma_{y} \\
\sigma_{z} \\
\tau_{y z} \\
\tau_{z x} \\
\tau_{x y}
\end{array}\right\}, \varepsilon=\left\{\begin{array}{l}
\varepsilon_{x} \\
\varepsilon_{y} \\
\varepsilon_{z} \\
\gamma_{y z} \\
\gamma_{z x} \\
\gamma_{x y}
\end{array}\right\}
$$

According to Hooke's law, equation (5) can be expressed as:

$$
\left\{\begin{array}{l}
\sigma_{x} \\
\sigma_{y} \\
\sigma_{z} \\
\tau_{y z} \\
\tau_{z x} \\
\tau_{x y}
\end{array}\right\}=\frac{E(1-v)}{(1+v)(1-2 v)}\left[\begin{array}{cccccc}
1 & \frac{v}{1-v} & \frac{v}{1-v} & 0 & 0 & 0 \\
1 & \frac{v}{1-v} & 0 & 0 & 0 \\
& 1 & 0 & 0 & 0
\end{array}\right]\left\{\begin{array}{l}
\varepsilon_{x} \\
\varepsilon_{y} \\
\varepsilon_{z} \\
\gamma_{y z} \\
\gamma_{z x} \\
\gamma_{x y}
\end{array}\right\}
$$

$E, v$ represent Young's modulus and is Poisson's ratio.

\section{Dispersion characteristics of Lamb wave in thin plate}

The phenomenon that the wave velocity changes with frequency is called frequency dispersion. In thin plate, Lamb wave whose velocity is determined by the frequency-thickness products has 
apparent dispersion characteristics. The velocity and mode of Lamb wave are correspond to a given frequency-thickness product. Fig. 2 shows Lamb wave dispersion curve in $8 \mathrm{~mm}$ thick titanium plate. There are some Lamb wave modes such as $A_{0} 、 S_{0} 、 A_{1} 、 S_{1} 、 A_{2} 、 S_{2}$, and the propagation velocity is related to the frequency if the thickness is constant.

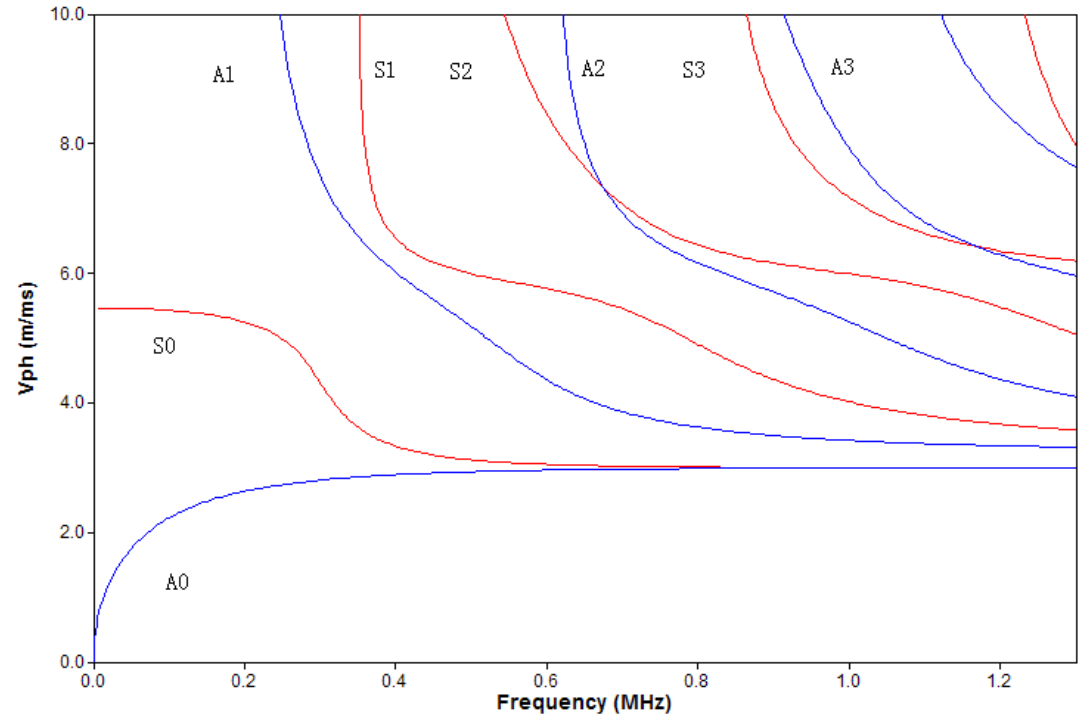

Fig.2 Lamb wave dispersion curves of titanium plate

\section{Simulation result}

\subsection{Single plate}

The key of FEM are establishing the Lamb wave propagation model, setting the boundary conditions and meshing. For single plate, it is advisable to take four node rectangular elements as the basic grids, and each node has two displacement degrees. In order to ensure the accuracy of calculation, the maximum size of the grid must satisfy the equation (8) [5]:

$\Delta x \leq \lambda_{\min } / 8$

$\Delta x, \lambda_{\min }$ represent the maximum element size and the minimum wavelength.

In order to ensure the stability of the algorithm, the time step must satisfy the equation (9):

$\delta t \leq 0.8 \frac{\Delta s}{c}$

$\Delta s, c$ represent the minimum element size and the wave velocity.

Fig.3 shows the finite element model of titanium plate, whose length is $800 \mathrm{~mm}$ and thickness $8 \mathrm{~mm}$. A 5 cycles Hanning window modulation sine single whose frequency is $100 \mathrm{KHz}$ is excited at point $\mathrm{A}$.

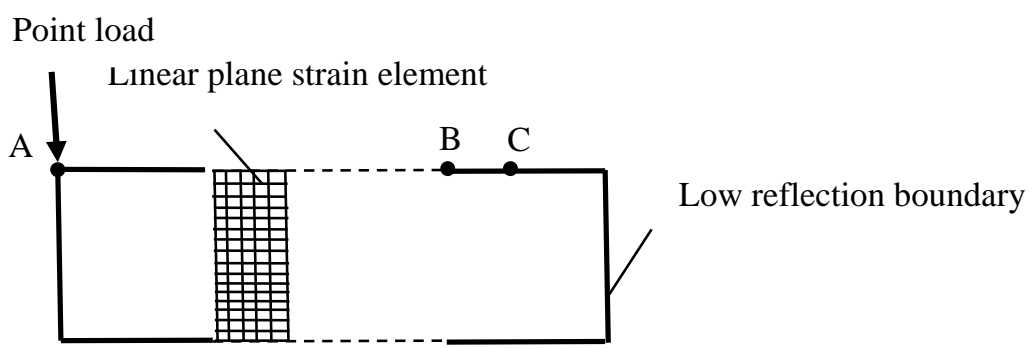

Fig. 3 Finite element model of titanium plate

In this solution, the total duration is $10^{-3} \mathrm{~s}$, the time step is $10^{-6} \mathrm{~s}$, the relative tolerance is $10^{-6}$, and the absolute tolerance is $10^{-20}$.

The finite element solution domain is usually a limited part, in which there is an artificial truncation boundary. The upper and lower surfaces are free boundaries, and the four side surfaces are low reflection boundaries. 
According to the total displacement of titanium plate, the time that the Lamb wave begins to be reflected is in $3.2 \times 10^{-4}$ seconds, and the time of the second reflection is in $6 \times 10^{-4}$ seconds. Considering the first reflection only, the two moments in $6 \times 10^{-5}$ seconds and in $3.6 \times 10^{-4}$ seconds are selected as the time before and after the reflection. It can be found that the displacement field is anti-symmetric, so the Lamb wave takes on A mode. As shown in Fig.4.

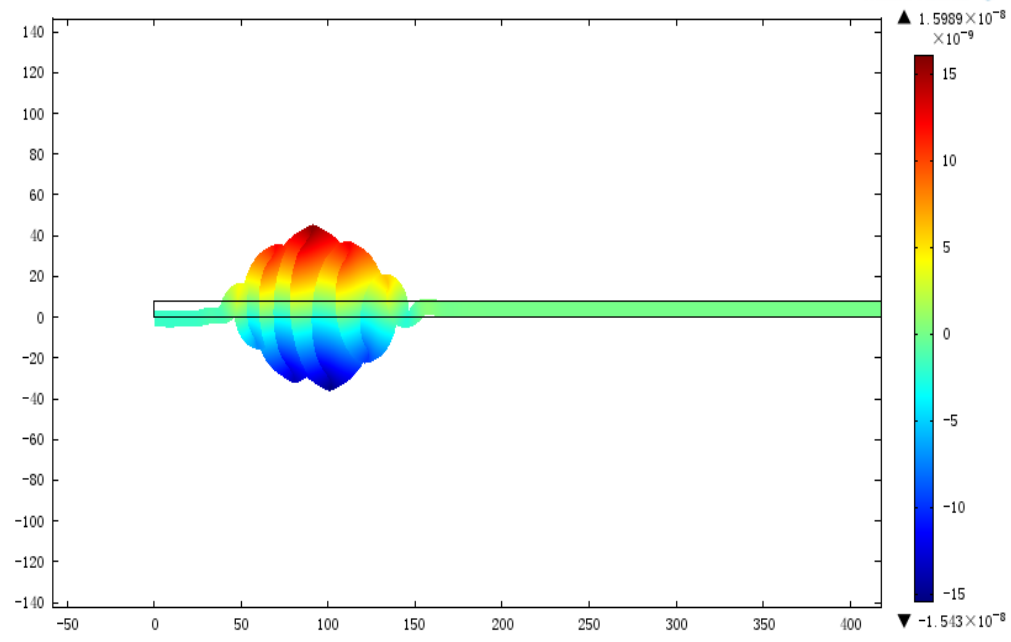

(a)6e-5s

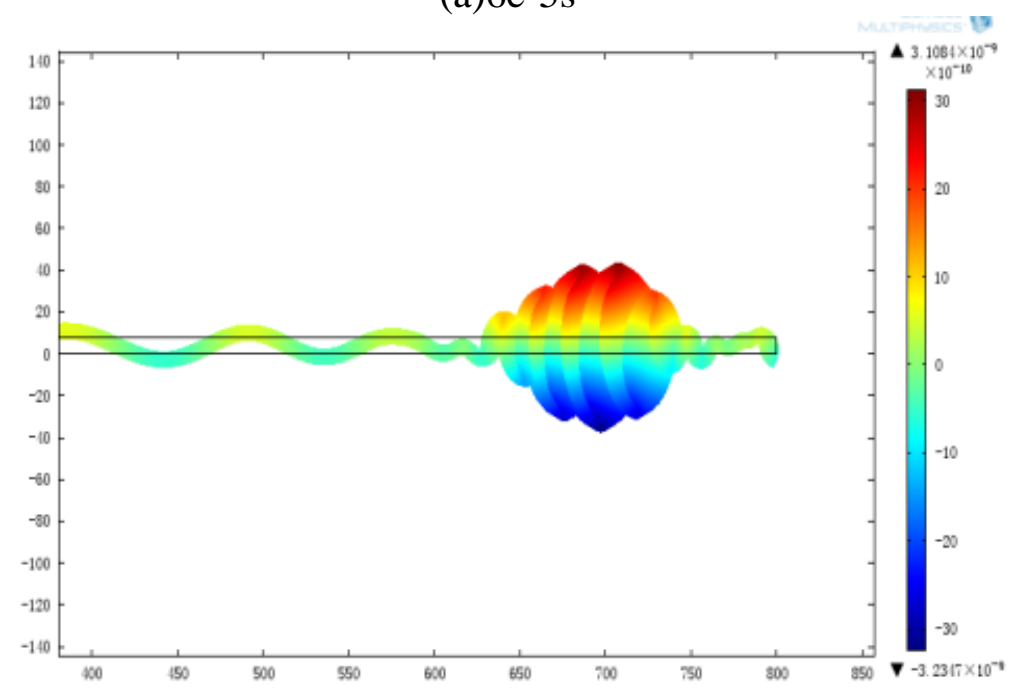

(b)3.6e-4s

Fig.4 Displacement field of Y direction

There are two monitoring points B and C. The distances from B to A and C to A are $500 \mathrm{~mm}$ and $600 \mathrm{~mm}$. The signals at the two points are shown in Fig. 5.

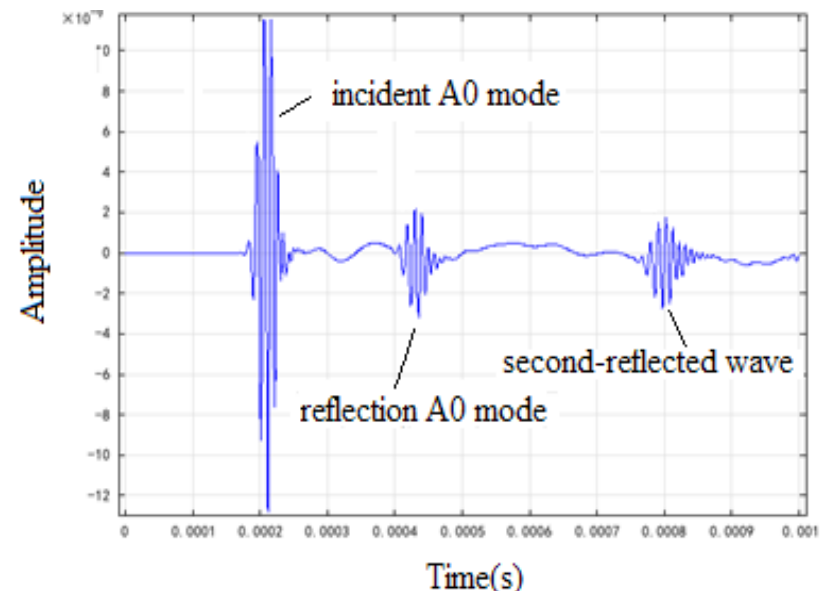

(a)Point B

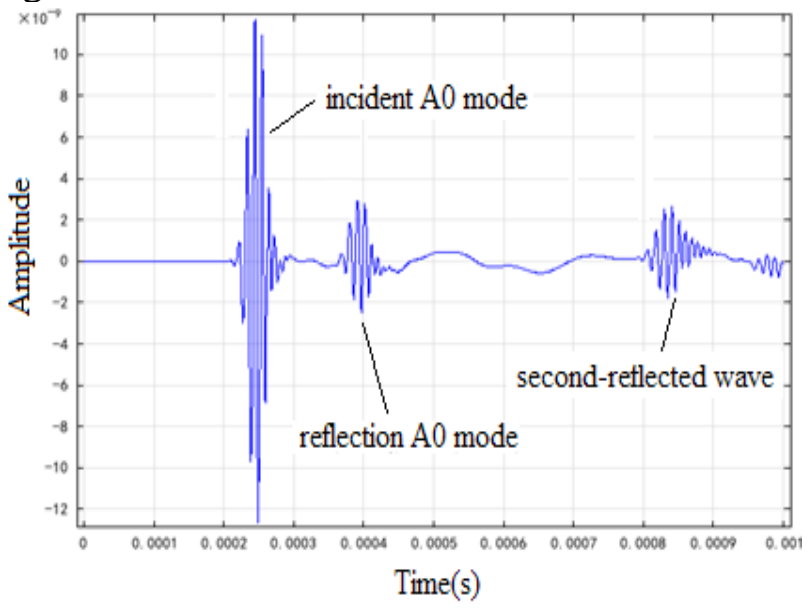

(b)Point C

Fig. 5 Two monitoring signals 
In Fig. 5(a), there are two wave packets which occur in $2.2 \times 10^{-4}$ seconds and $4.3 \times 10^{-4}$ seconds. The Lamb wave begins to be reflected in $3.2 \times 10^{-4}$ seconds, so the second packet is the reflected wave of the first one, and whose velocity is:

$$
c_{g}=\frac{500 \times 10^{-3}}{2.2 \times 10^{-4}}=2272.72 \mathrm{~m} / \mathrm{s}
$$

According to the dispersion curve in Fig.2, the velocity of $A_{0}$ mode Lamb wave at $100 \mathrm{KHz}$ is $2241.95 \mathrm{~m} / \mathrm{s}$, and the error is 1.3\%. This conclusion also can be obtained in Fig.5 (b).

\subsection{Bilayer plate}

Assuming the upper layer material is plexiglass whose thickness is $10 \mathrm{~mm}$, and the lower layer material is steel whose thickness is $4 \mathrm{~mm}$, as shown in Fig.6. A sine signal whose frequency is $5 \mathrm{MHz}$ is excited on the upper surface of the bilayer plate.

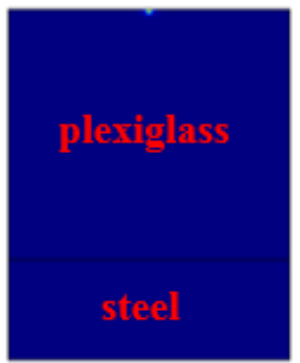

(a) $0.1 \mu \mathrm{s}$

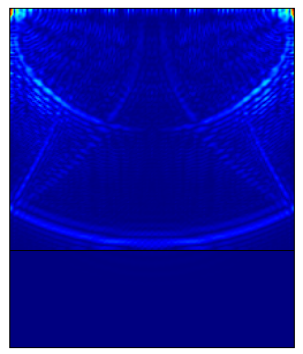

(e) $3.6 \mu \mathrm{s}$

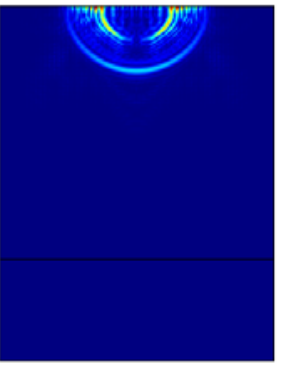

(b) $1 \mu \mathrm{s}$

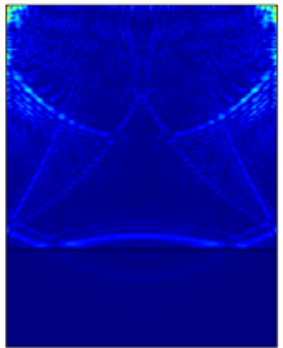

(f) $3.9 \mu \mathrm{s}$

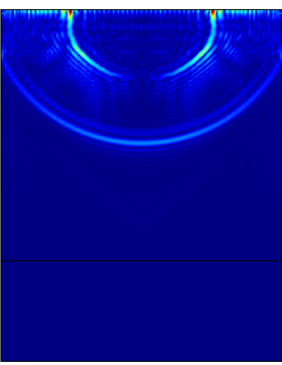

(c) $2 \mu \mathrm{s}$

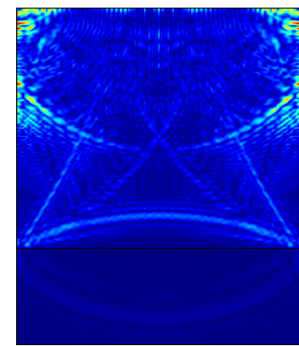

(g) $4.2 \mu \mathrm{s}$

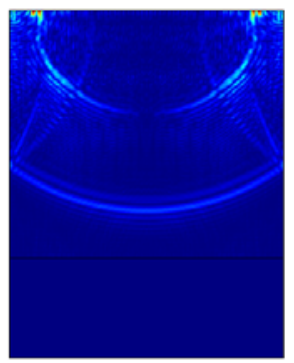

(d) $3 \mu s$

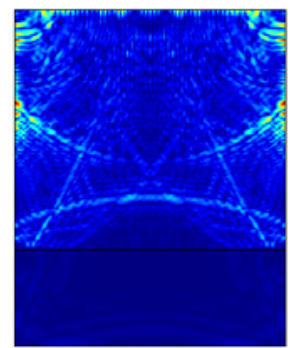

(h) $4.5 \mu \mathrm{s}$

Fig.6 Sound field in different time

The Fig. 6 shows the sound field in different time. In $0.1 \mu \mathrm{s}$, a point load occurs on the upper surface; in $1 \mu \mathrm{s}$, longitudinal and transverse wave began to separate, and the longitudinal is in front; in $2 \mu \mathrm{s}$ and $3 \mu \mathrm{s}$, the ultrasonic began to be reflected on the side surfaces; in 3.6 $\mu \mathrm{s}$, the longitudinal wave arrives the interface between the two mediums; in $3.9 \mu \mathrm{s}$, part of the ultrasonic is reflected in the plexiglass, and the other is incident to the steel; in $4.2 \mu \mathrm{s}$, ultrasonic is out of the interface completely; in $4.5 \mu \mathrm{s}$, there are multiple reflections and mode conversions.

\section{Conclusions}

In this paper, FEM is used to simulate the propagation process of Lamb wave in thin plate. The Lamb wave mode can be judged from the displacement field, and the physical meaning of the wave packets can be analyzed by the arrival time. The velocity error of numerical results is very small, which proves the validity of the numerical results. The incident and reflection of longitudinal and transverse waves are reproduced in bilayer plate. So this work can supply guidance for Lamb wave testing in engineering practice.

\section{References}

[1] Xiaohuai Ma. Noise analysis during ultrasonic testing of titanium alloys [J]. Nondestructive testing, 2006, 26(12): 649-651. 
[2] Dean Ta, Ruiju Huang, Zhenqing Liu. Application of numerical analysis in ultrasonic testing [J].Nondestructive testing, 2001, 23(11): 485-488.

[3] Jianzhong Chen, Yaowu Shi. Numerical simulation of ultrasonic testing [J].Nondestructive testing, 2001, 23(5):198-201.

[4] Zhenpeng Liao. Numerical simulation of near field fluctuation [J].Advances in mechanics, 1997, 27(2):193- 216.

[5] Hui Ding. Computational ultrasound [M], 2010, Beijing: Science Press. 